\title{
Overexpression of the pituitary tumor transforming gene upregulates metastasis in malignant neoplasms of the human salivary glands
}

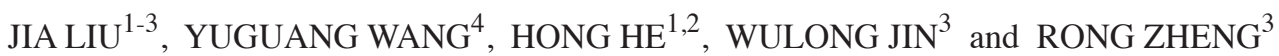

\author{
${ }^{1}$ The State Key Laboratory Breeding Base of Basic Science of Stomatology (Hubei-MOST) \& Key Laboratory of Oral \\ Biomedicine Ministry of Education; ${ }^{2}$ Department of Orthodontics, School and Hospital of Stomatology, Wuhan University, \\ Wuhan, Hubei 430079; ${ }^{3}$ Department of Stomatology, The Affiliated Hospital of Inner Mongolia Medical University, \\ Hohhot, Inner Mongolia 010050; ${ }^{4}$ Department of Diagnostic Imaging, The Second Affiliated Hospital, \\ Inner Mongolia Medical University, Hohhot, Inner Mongolia 010030, P.R. China
}

Received March 10, 2015; Accepted June 1, 2015

DOI: $10.3892 / \mathrm{etm} .2015 .2566$

\begin{abstract}
Salivary gland malignant neoplasms (SGMNs) represent a group of malignant solid tumors with heterogeneity in their cellular make-up, which causes difficulty with regard to the immunohistochemical confirmation of their cytological features. In the present study, overexpression of the pituitary tumor transforming gene (PTTG) was evaluated in human mucoepidermoid carcinoma specimens with a submaxillary salivary gland origin by immunohistochemical analysis, western blot analysis and reverse transcription quantitative polymerase chain reaction. In addition, a SGMN cell line was constructed, namely A-253 PTTG (+), which overexpressed PTTG. Subsequently, the regulatory role of PTTG in the proliferation and migration of A-253 cells was investigated. The immunohistochemical results demonstrated that there was a higher rate of PTTG-positive cells in the SGMN tissues when compared with the control submaxillary salivary gland tissues. Furthermore, PTTG expression at a mRNA and protein level was significantly higher in the SGMN specimens when compared with the control specimens. In addition, the rates of proliferation and migration of the A-253 PTTG (+) cells were significantly higher compared with the A-253 PTTG (-) cells. Therefore, PTTG was demonstrated to play an important role in SGMN cell proliferation and migration, and may subsequently be a notable marker for SGMN diagnosis and a potential target for anticancer therapy.
\end{abstract}

Correspondence to: Professor Hong He, Department of Orthodontics, School and Hospital of Stomatology, Wuhan University, 237 Luo Yu Road, Hongshan, Wuhan, Hubei 430079, P.R. China

E-mail: drhehong@163.com

Key words: pituitary tumor transforming gene, proliferation, metastasis, salivary glands malignant neoplasm

\section{Introduction}

Salivary gland malignant neoplasms (SGMNs) are a group of malignant solid tumors that present favorable features with regard to locoregional invasion and metastasis (1). SGMNs account for 3-6\% of all head and neck cancers (2), with an estimated global morbidity rate ranging between 0.4 and 2.6 cases per 100,000 individuals (3). In modern pathology, SGMNs are one of the major diagnostic challenges due to their heterogeneity in cellular make-up, which poses difficulty for the immunohistochemical confirmation of their cytological features (4). However, the SGMN classification system continues to be based on their histopathological features, biological behavior and histogenesis (5). Previous studies have demonstrated that salivary gland carcinomas exhibit overexpression of cyclin-dependent kinase inhibitor $2 \mathrm{~A}(C D K N 2 A)$, $T P 53$ and epidermal growth factor (EGFR) (6-8). In addition, the frequency of EGFR-positive mucoepidermoid carcinomas was $82-91 \%$ in SGMN samples $(7,9)$. These observations indicate that aberrant expression of cell cycle factors and EGFR in a subfraction of tumor cells refers to deregulated cell homeostasis.

The pituitary tumor transforming gene (PTTG) has been identified as an oncogene, inducing cellular transformation in vitro and tumor formation in nude mice (10). PTTG encodes human securin, which participates in the mitotic spindle checkpoint pathway and inhibits sister chromatid separation to ensure chromosomal stability $(11,12)$. Although PTTG expression is restricted in normal tissue, PTTG has been shown to be abundantly expressed in SGMNs (13) and other non-endocrine-associated cancers, such as colon (13), gastric (14), lung (15) and esophagus cancer (16). In particular, PTTG has been recognized as one of the key 'signature genes' to predict metastasis in primary adenocarcinomas of the lung, breast and prostate, as well as medulloblastomas (17). Although PTTG overexpression correlates with metastasis and poor overall survival times in these cancer types (18), whether the oncogenic molecule contributes to the tumorigenesis of SGMNs remains unclear. 
In the present study, PTTG overexpression was evaluated in human mucoepidermoid carcinoma specimens from the submaxillary salivary gland using immunohistochemical analysis and western blot analysis. In addition, to investigate the oncogenic role of PTTG in SGMNs, a cell line was constructed that overexpressed PTTG and coexpressed an enhanced green fluorescence protein (EGFP) marker. The A-253 cell line was an epidermoid carcinoma cell line originating from the human submaxillary salivary gland. Subsequently, the influence of PTTG on the proliferation and migration rates of A-253 cells was investigated. The role of PTTG in SGMN cell migration was investigated with the aim of assessing the potential of PTTG as a target for anticancer therapy.

\section{Materials and methods}

Submaxillary SGMN specimens, cell culture and construction of PTTG-overexpressing A-253 cells. The study was approved by the Medical Ethics Committee of the Affiliated Hospital of Inner Mongolia Medical University (Hohhot, China). In total, 19 human mucoepidermoid carcinoma specimens of the submaxillary salivary gland and 18 control submaxillary salivary gland specimens were obtained by surgical resection prior to the administration of radiotherapy or chemotherapy for the SGMN patients or pleomorphic adenoma patients. Informed consent and agreement were provided by all the patients. Clinicopathological data of the patients were recorded prospectively, including the age at diagnosis, tumor size, axillary lymph node metastasis and histological grade. All fresh tumor specimens were immediately frozen in liquid nitrogen and stored at $-70^{\circ} \mathrm{C}$ following resection. An epidermoid carcinoma cell line originating from the human submaxillary salivary gland (A-253 cell line) was purchased from the American Type Culture Collection (Rockville, MD, USA). The cells were cultured at $37^{\circ} \mathrm{C}$ in a humidified atmosphere of $5 \% \mathrm{CO}_{2}$ in McCoy's 5a Medium Modified (Sigma-Aldrich, St. Louis, MO, USA), supplemented with $10 \%$ fetal bovine serum (Gibco Life Technologies, Rockville, MD, USA).

To generate an A-253 cell line that overexpressed PTTG, the wild-type PTTG coding sequence was amplified and cloned into a pcDNA3.1 (+) vector (Invitrogen Life Technologies, Carlsbad, CA, USA). Subsequently, the PTTG-2A-EGFP-pcDNA3.1 (+) or control CAT-pcDNA3.1 (+) vectors were transfected into the A-253 cells using Lipofectamine 2000 (Invitrogen Life Technologies). The PTTG- and EGFP-positive cell clones were selected under the pressure of $1.5 \mathrm{mg} / \mathrm{ml} \mathrm{G} 418$ (Sigma-Aldrich), and maintained in medium containing $1 \mathrm{mg} / \mathrm{ml} \mathrm{G} 418$.

Immunohistochemical staining. SGMN tissue slides were successively deparaffinized by heating at $55^{\circ} \mathrm{C}$ for $30 \mathrm{~min}$, and rehydrated serially in 100, 90 and $70 \%$ ethanol, and phosphate-buffered saline (PBS). Subsequently, antigen retrieval was performed by heating for $20 \mathrm{~min}$ at $98^{\circ} \mathrm{C}$ in $10 \mathrm{mM}$ sodium citrate ( $\mathrm{pH}$ 6.0), after which the endogenous peroxidase activity was blocked by incubation with $0.3 \%$ hydrogen peroxide for $20 \mathrm{~min}$. Rabbit polyclonal antibodies targeting human PTTG (1:100; PA5-14240) and $\beta$-actin (1:300; PA1-183; Thermo Fisher Scientific, Waltham, MA, USA) were utilized to stain for PTTG and $\beta$-actin expression. The samples were incubated with the primary antibodies against PTTG and $\beta$-actin for $1 \mathrm{~h}$ at room temperature, followed by incubation with a goat anti-rabbit/mouse $\mathrm{IgG}$ horseradish peroxidase (HRP)-conjugated secondary antibody $(1: 1,000$; ab6721; Abcam, Cambridge, UK) at room temperature for 1 h. Subsequently, the HRP substrate, 3,3'-diaminobenzidene (Abcam), was added for 1-5 min and counterstained with Mayer's hematoxylin, after which the samples were dehydrated and sealed with cover slips.

RNA isolation and reverse transcription quantitative polymerase chain reaction $(R T-q P C R)$. Total cellular mRNA was isolated from the tumor specimens or from the A-253 cells using TRIzol reagent (Invitrogen Life Technologies), according to the manufacturer's instructions. RT-qPCR was performed to quantify the mRNA expression levels of PTTG using a SYBR PrimeScript RT-qPCR kit (Takara Bio, Inc., Tokyo, Japan) and a LightCycler 2.0 (Roche Diagnostics Gmbh, Mannheim, Germany), where $\beta$-actin was used as an internal control. The primer sequences for PTTG were as follows: 5'-ACC TTT GCT TCT CCC ACC TT-3' (sense) and 5'-CAAATA CAC ACA AAC TCT GAA GCA-3' (antisense). The primer sequences for $\beta$-actin were as follows: 5'-TTC TAC AAT GAG CTG CGT GTG-3' (sense) and 5'-GGG GTG TTG AAG GTC TCA AA-3' (antisense). All the primers were synthesized by Shanghai Sangon Biological Engineering Technology \& Services Co., Ltd. (Shanghai, China). PTTG expression was normalized against $\beta$-actin, and was expressed as the fold change over the control, as calculated according to the $\Delta \Delta \mathrm{Ct}$ method (19).

Western blot analysis for PTTG expression. PTTG protein expression levels in the tumor specimens were analyzed by western blot analysis. The tumor specimens were homogenized prior to protein isolation. The homogenized specimens or cultured cells were collected and lysed with a lysis reagent (Pierce Biotechnology, Inc., Rockford, IL, USA), according to the manufacturer's instructions, which was supplemented with a protease inhibitor cocktail (Thermo Fisher Scientific). Protein samples were separated by $12 \%$ SDS-PAGE and transferred to a nitrocellulose membrane (EMD Millipore, Bedford, MA, USA). The protein expression levels of PTTG and $\beta$-actin were quantified via successive incubation at $37^{\circ} \mathrm{C}$ for $1 \mathrm{~h}$ with anti-PTTG (1:300; PA5-14240) or anti- $\beta$-actin $(1: 1,00$; PA1-183) polyclonal rabbit primary antibodies, followed by a monoclonal peroxidase-conjugated secondary antibody against rabbit IgG (1:1,000; A1949; Sigma-Aldrich). An electrochemiluminescence detection system (GE Healthcare Life Sciences, Uppsala, Sweden) was used for visualization of the membranes, according to the manufacturer's instructions.

Cell count assay and cell migration assay. A cell count assay was performed as previously described (20). Briefly, $1 \times 10^{3}$ or 1x10 4 A-253 PTTG (+) and A-253 PTTG (-) cells/ml were seeded in six-well plates and incubated at $37^{\circ} \mathrm{C}$ for 12,24 or $48 \mathrm{~h}$, or for 1, 2 or 4 days, after which the cells were trypsinized. The number of viable cells was counted in a hemocytometer (Tainuokeji, Tai'an, China) with the use of trypan blue staining (Santa Cruz Biotechnology, Inc., Dallas, TX, USA). The rate of cell migration was determined by a scratch assay. A-253 PTTG 

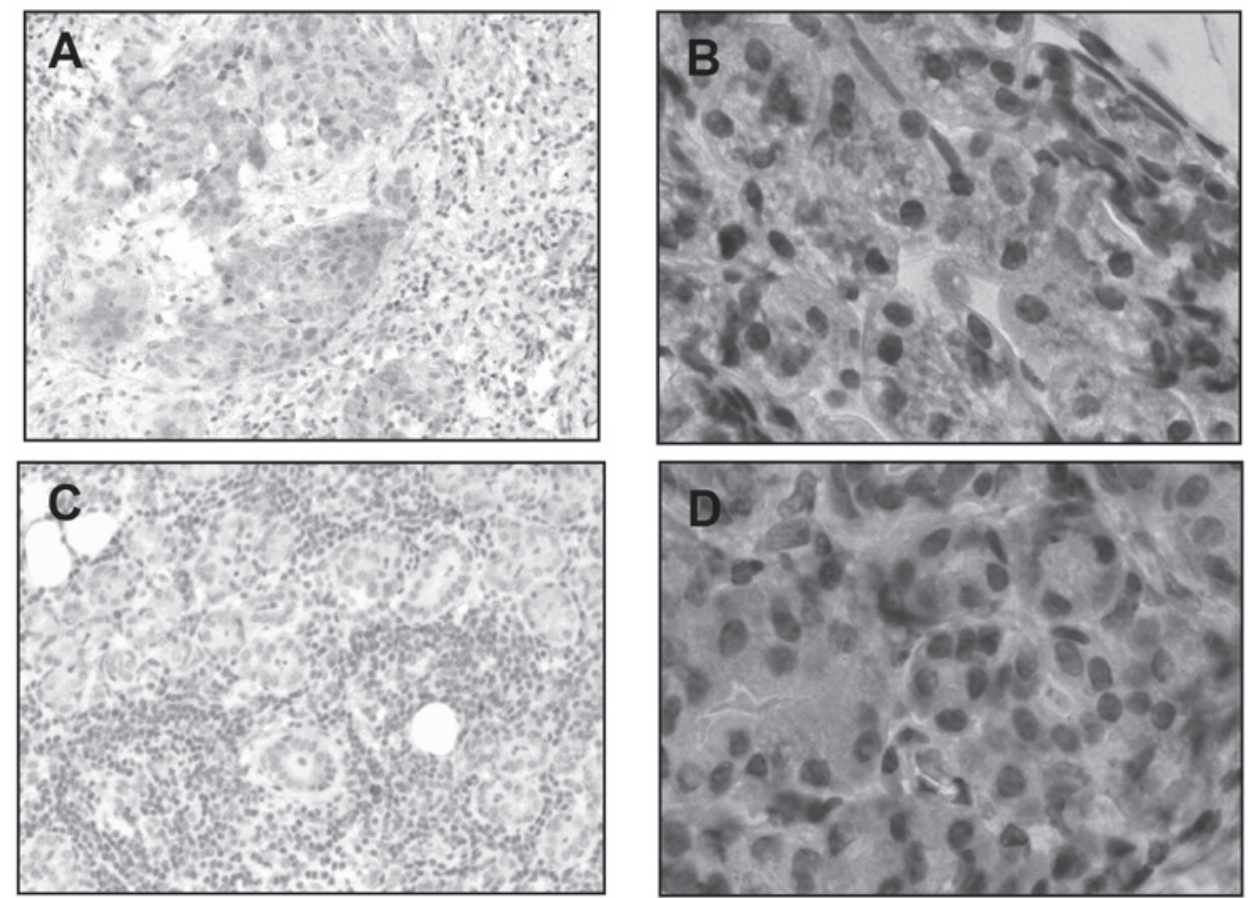

Figure 1. Typical immunohistochemical staining for pituitary tumor transforming gene (PTTG) expression in malignant neoplasms of the human salivary glands. (A and B) Representative immunohistochemical staining showing the expression of PTTG in tissues collected from malignant neoplasms of the human salivary gland (positive samples, 14/19; magnification, x100 and x800, respectively). (C and D) Representative immunohistochemical staining showing the expression of PTTG in control human salivary gland tissues (positive samples, 8/18; magnification, x100 and x800, respectively).

(+) or A-253 PTTG (-) cells were cultivated to $90 \%$ confluence on six-well plates. Subsequently, cell scrapers (Corning, Inc., Corning, NY, USA) were utilized to scratch the confluent cells. After $48 \mathrm{~h}$, the cells that had migrated across the baseline were observed and counted using a BX60 microscope (Olympus Optical Co., Ltd., Tokyo, Japan). All the experiments were repeated in triplicate.

Statistical analysis. Statistical analysis was performed using GraphPad Prism 5 software (GraphPad Software, Inc., La Jolla, CA, USA). All data are expressed as the mean \pm standard error of the mean. The difference in the PTTG-positivity rate between the two groups was evaluated using the $\chi^{2}$ test. Comparisons between the expression of PTTG at an mRNA or protein level, or the cell number in the two groups, were analyzed using the Student's t-test. $\mathrm{P}<0.05$ was considered to indicate a statistically significant difference.

\section{Results}

Overexpression of PTTG in the SGMN specimens. To identify a possible oncogenic role of PTTG in SGMNs, the PTTG expression levels in the SGMN specimens were determined. Firstly, as shown in Fig. 1A and B, there was a higher rate of PTTG-positive cells in the SGMN tissues when compared with the control submaxillary salivary gland tissues (14/19 vs. $7 / 18, \mathrm{P}=0.0327$ ), with a cutoff of 2 positive cells per field. To further reveal the difference in the PTTG expression levels between the tumor and non-tumor groups, the mRNA expression levels of PTTG in all the specimens from the two groups were analyzed. The results demonstrated that the mRNA expression level of PTTG in the SGMN specimens
A

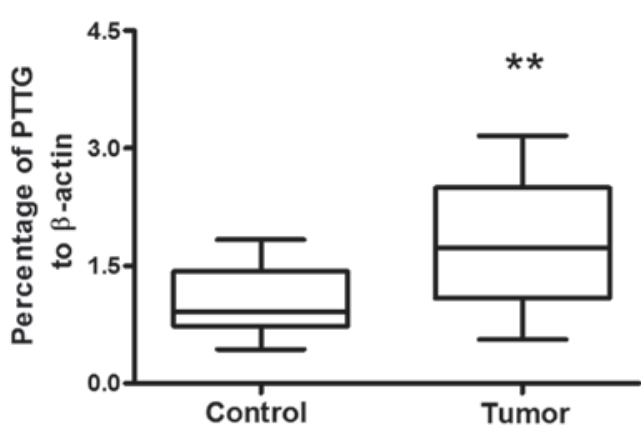

B

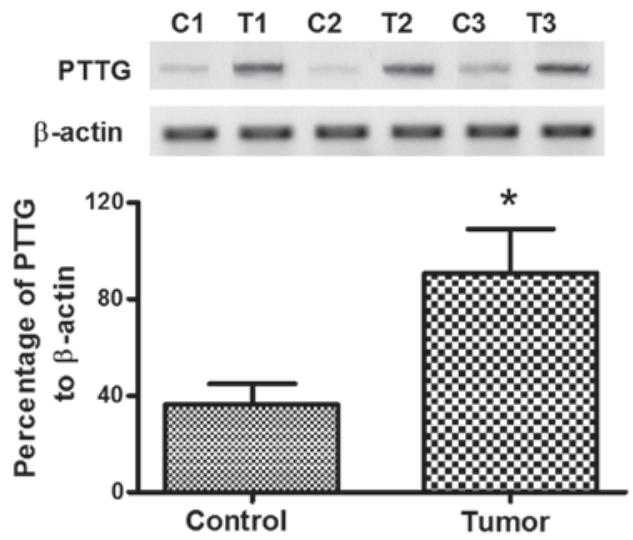

Figure 2. PTTG overexpression at an mRNA and protein level in malignant neoplasms of the human salivary glands. (A) Relative mRNA expression levels of PTTG in the tissues collected from malignant neoplasms of the human salivary gland $(n=19)$ or control human salivary gland tissues $(n=18)$, as determined by quantitative polymerase chain reaction. (B) Overexpression of PTTG at a protein level in the tissues collected from malignant neoplasms of the human salivary gland $(\mathrm{n}=16)$ or control human salivary gland tissues $(\mathrm{n}=14)$, as determined by western blot analysis. ${ }^{*} \mathrm{P}<0.05$ and ${ }^{* *} \mathrm{P}<0.01$, vs. control. PTTG, pituitary tumor transforming gene. 
A

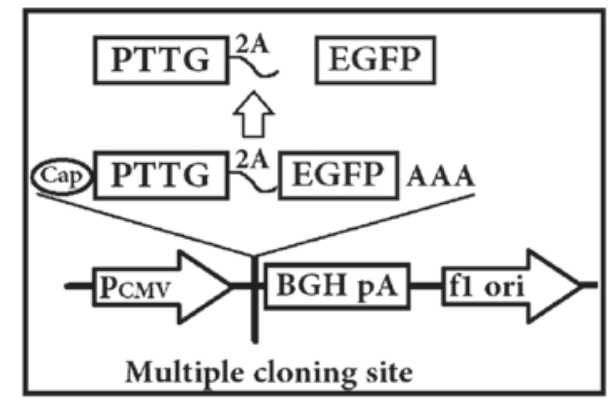

C

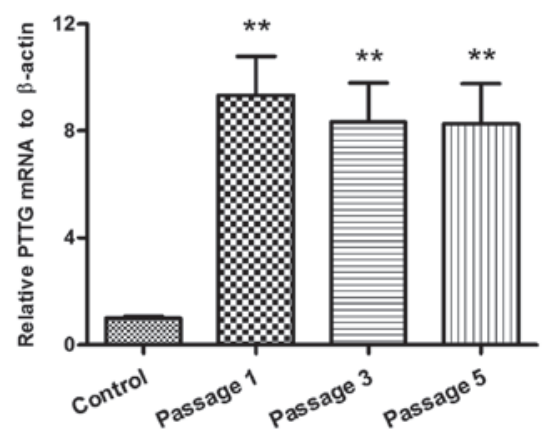

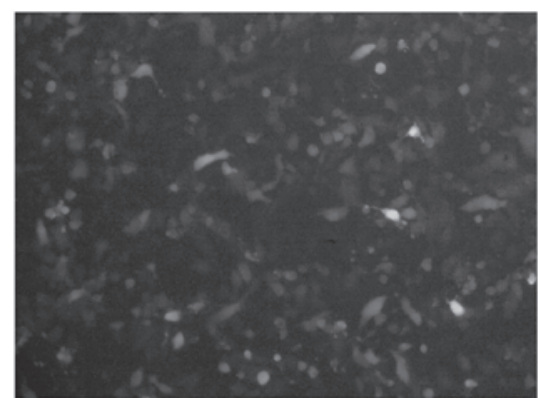

D
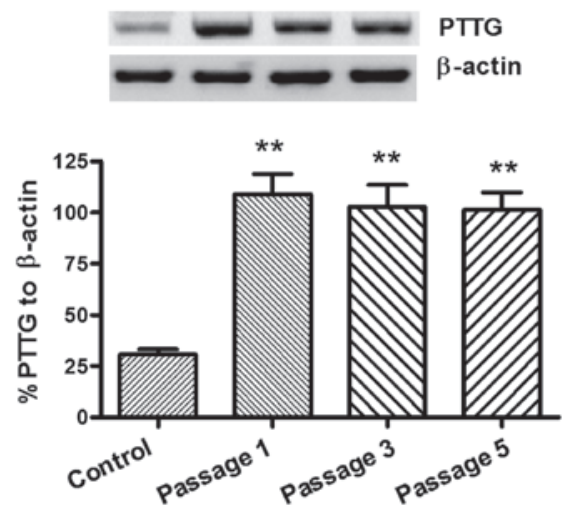

Figure 3. Construction of an A-253 cell line that overexpressed PTTG. (A) Schematic diagram of the plasmid coexpressing PTTG and EGFP with a 2A peptide sequence. (B) Selection of PTTG-positive A-253 cells (also EGFP-positive). (C) PTTG expression at an mRNA level in the PTTG-positive A-253 cells. (D) PTTG expression at a protein level in the PTTG-positive A-253 cells. Results of the PTTG expression levels at an mRNA and protein level were averaged for three independent experiments, ${ }^{* *} \mathrm{P}<0.05$, vs. control. PTTG, pituitary tumor transforming gene; EGFP, enhanced green fluorescent protein.

A

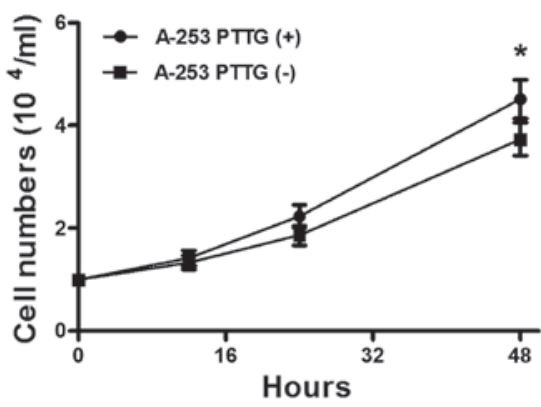

C Day 0
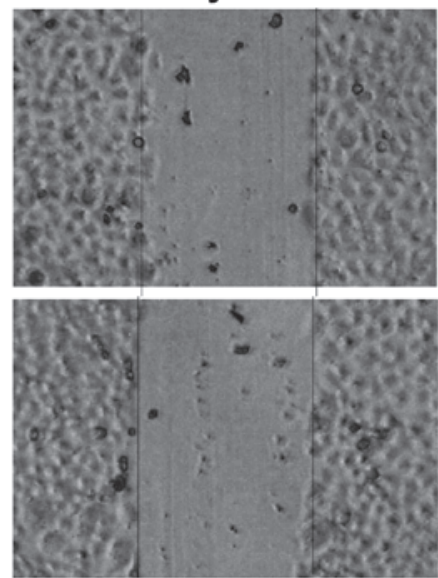

Day 2

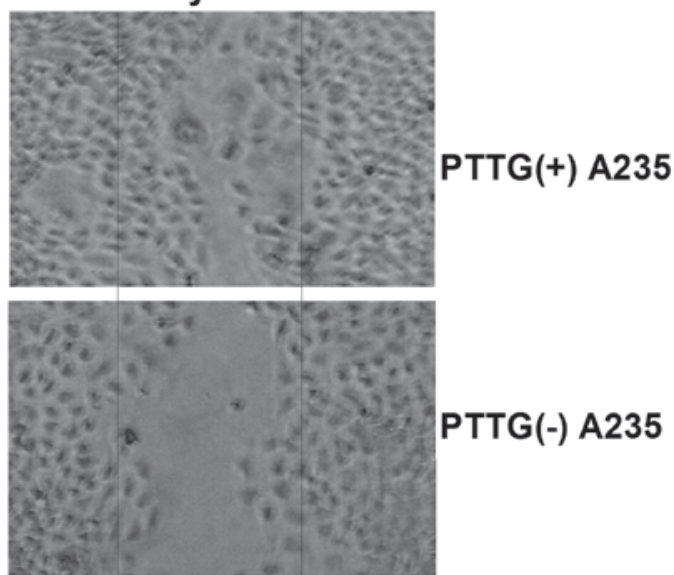

B

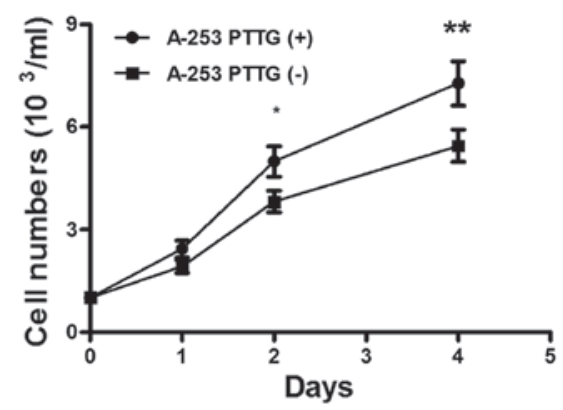

D

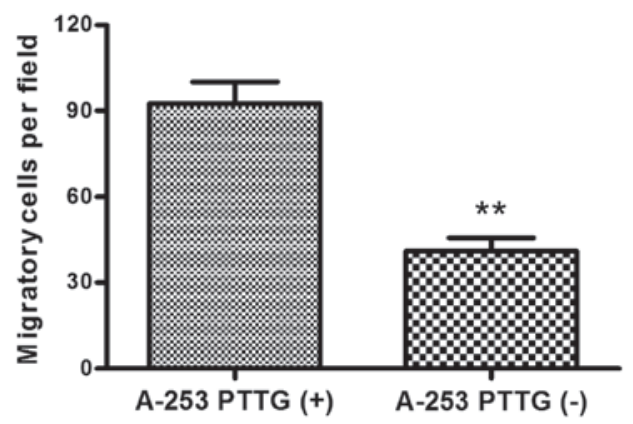

Figure 4. Overexpression of PTTG upregulates the growth and migration of A-253 cells. A significantly higher growth rate was observed in the A-253 PTTG (+) cell cultures when compared with the A-253 PTTG (-) cells following an initial inoculation at (A) $10^{4} \mathrm{cell} / \mathrm{s} / \mathrm{ml}$ and (B) $10^{3}$ cells $/ \mathrm{ml}$. (C) Representative images showing the migration of the A-253 PTTG (+) and A-253 PTTG (-) cells. (D) Quantification of the A-253 PTTG (+) cells and A-253 PTTG (-) cells that had migrated following inoculation for 4 days, as determined by a cell scratch assay. Separate experiments were performed in triplicate. " $\mathrm{P}<0.05$ and ${ }^{* *} \mathrm{P}<0.01$, A-253 PTTG (+) cells vs. A-253 PTTG (-) cells. PTTG, pituitary tumor transforming gene. 
was $1.772 \pm 0.187(n=19)$, as compared with the average level of $1.032 \pm 0.096(n=18)$ in the control group, which was a statistically significant difference ( $\mathrm{P}=0.0014$; Fig. 2A). In addition, the protein expression levels of PTTG were further analyzed using western blot analysis in 16 SGMN specimens and 14 control specimens that were of sufficient sample volume to facilitate the assay. As shown in Fig. 2B, the protein expression level of PTTG in the SGMN specimens was also significantly higher when compared with the control specimens $(\mathrm{P}<0.05$, paired-samples t-test). Therefore, the overexpression of PTTG in SGMN specimens was confirmed.

Construction of an A-253 stable cell line overexpressing PTTG. To identify the oncogenic role of PTTG in SGMN cells, a PTTG and EGFP coexpressing A-253 cell line was constructed. As shown in Fig. 3A, the cDNA of PTTG and EGFP were linked by a $2 \mathrm{~A}$ peptide coding sequence, which guaranteed the transcription of PTTG and EGFP in one mRNA molecule, but enabled the translation of two separate proteins $(21,22)$. EGFP-positive A-253 cells, which were transfected with the recombinant PTTG-2A-EGFP-pcDAN3.1 (+) plasmid, were selected under G418 pressure. Following serial passages with $1.5 \mathrm{mg} / \mathrm{ml} \mathrm{G} 418,>85 \%$ of the A-253 cells were determined to be EGFP-positive (Fig. 3B). Subsequently, the overexpression of PTTG was determined in the EGFP-positive A-253 cells. Stabilized PTTG overexpression at the mRNA level in the A-253 PTTG (+) cells was determined using a RT-qPCR method with the PTTG specific primers. Significantly higher expression levels of PTTG mRNA were observed in the A-253 PTTG (+) cells at the passages of 1,3 or $5(\mathrm{P}<0.01$, vs. control A-253 cells, using the t-test; Fig. 3C). In addition, PTTG overexpression at a protein level was stabilized in the A-253 PTTG $(+)$ cells $(\mathrm{P}<0.01$, vs. control A-253 cells, as determined with the t-test; Fig. 3D). Therefore, the A-253 PTTG (+) cells were shown to stably express PTTG.

PTTG overexpression promotes the growth and migration of A-253 cells. The influence of PTTG overexpression on the growth of SGMN cells was subsequently evaluated. The growth of the A-253 PTTG (+) or A-253 PTTG (-) cells in vitro was assessed using a cell count assay, in which cell counting was conducted at 12, 24 and $48 \mathrm{~h}$ after cell inoculation. As shown in Fig. 4A, when inoculated at a density of $10^{4}$ cells $/ \mathrm{ml}$, the A-253 PTTG (+) cells grew more efficiently compared with the A-253 PTTG (-) cells at $48 \mathrm{~h}$ after cell inoculation, and the difference was statistically significant $\left(4.51 \pm 0.38 \times 10^{4} / \mathrm{ml}\right.$ for A-253 PTTG (+) cells and $3.733 \pm 0.32 \times 10^{4} / \mathrm{ml}$ for A-253 PTTG (-) cells at $48 \mathrm{~h}$ after inoculation; $\mathrm{P}<0.05)$. In addition, the difference in growth between the two cell lines was reconfirmed following inoculation at $10^{3}$ cells $/ \mathrm{ml}$, where the growth of the A-253 PTTG (+) cells was also significantly more efficient compared with the A-253 PTTG (-) cells (Fig. 4B; P<0.05 for $24 \mathrm{~h}$; $\mathrm{P}<0.01$ for $48 \mathrm{~h}$ ). Thus, the promotive role of PTTG in the growth of SGMN cells was confirmed. Cell migration contributes to the extent of tumor metastasis; thus, the migration of the A-253 PTTG (+) and A-253 PTTG (-) cells were further assessed using a scratch assay. As shown in Fig. 4B and C, at $48 \mathrm{~h}$ after the initiation of the scratch assay, a significantly increased number of A-253 PTTG (+) cells had migrated across the baseline when compared with the A-253 PTTG (-) cells $(92.67 \pm 7.51$ vs. $41 \pm 4.62, \mathrm{P}<0.01)$. Therefore, the results indicated that overexpression of PTTG stimulated the growth and migration of SGMN cells.

\section{Discussion}

In addition to the low incidence of SGMNs, the heterogeneity of their cellular make-up poses difficulty for the immunohistochemical confirmation of their cytological features (4). In addition, SGMNs are a major diagnostic challenge since the SGMN classification system is based on histopathological features, biological behavior and histogenesis (5). In previous years, a number of studies have recognized several molecular markers, such as CDKN2A, TP53 and EGFR, in SGMNs (6-8). The aberrant expression of these markers has been shown to significantly correlate with the malignancy of neoplasms in the salivary glands. To the best of our knowledge, the present study has demonstrated for the first time that PTTG is overexpressed in SGMN specimens. Furthermore, the overexpression of PTTG was repeatedly confirmed at a protein level by immunohistochemical analysis and western blot analysis, and at an mRNA level by RT-qPCR analysis. Therefore, PTTG overexpression is associated with SGMNs.

PTTG is frequently upregulated in human tumors and is known to be involved in metastasis. Increasing evidence reveals a correlation between PTTG overexpression and metastasis in various types of cancer, including colon (23), breast (13), gastric (24), and prostate cancer (25). However, the oncogenic role of PTTG in SGMNs remains unclear. In the present study, an A-253 stable cell line that overexpressed PTTG was constructed using a $2 \mathrm{~A}$ peptide coexpressing method (21,22). Simultaneous expression of EGFP with PTTG guaranteed the simplicity of PTTG-positivity selection. The EGFP-positive A-253 PTTG (+) cells were shown to stably overexpress PTTG at an mRNA and protein level. Further investigations demonstrated that the overexpression of PTTG significantly promoted A-253 cell proliferation and migration, as demonstrated by the cell count assay and cell migration assay. However, the detailed mechanisms underlying the PTTG-induced promotion of cell proliferation and migration remain unknown; thus, further investigation is required.

In conclusion, the present study identified PTTG overexpression in human mucoepidermoid carcinomas of the submaxillary salivary gland. In addition, PTTG was confirmed to significantly promote SGMN cell proliferation and migration in A-253 cells overexpressing PTTG and coexpressing EGFP; a cell line that was first constructed in this study. Therefore, the observations of the present study indicate that PTTG plays an important role in SGMN cell migration, and may subsequently be a notable marker for SGMN diagnosis and a potential target for anticancer therapy.

\section{Acknowledgements}

The study was supported by a grant from the Affiliated Hospital of Inner Mongolia Medical University (no. 201207).

\section{References}

1. Lopes MA, Santos GC and Kowalski LP: Multivariate survival analysis of 128 cases of oral cavity minor salivary gland carcinomas. Head Neck 20: 699-706, 1998. 
2. Hocwald E, Korkmaz H, Yoo GH, Adsay V, Shibuya TY, Abrams J and Jacobs JR: Prognostic factors in major salivary gland cancer. Laryngoscope 111: 1434-1439, 2001.

3. Speight PM and Barrett AW: Prognostic factors in malignant tumours of the salivary glands. Br J Oral Maxillofac Surg 47: 587-593, 2009.

4. Rapidis AD, Givalos N, Gakiopoulou H, Stavrianos SD, Faratzis G Lagogiannis GA, Katsilieris I and Patsouris E: Mucoepidermoid carcinoma of the salivary glands. Review of the literature and clinicopathological analysis of 18 patients. Oral Oncol 43: 130-136, 2007.

5. Thompson L: World Health Organization classification of tumours: Pathology and genetics of head and neck tumours. Ear Nose Throat J 85: 74, 2006.

6. Etges A, Nunes FD, Ribeiro KC and Araújo VC: Immunohistochemical expression of retinoblastoma pathway proteins in normal salivary glands and in salivary gland tumours. Oral Oncol 40: 326-331, 2004.

7. Shang J, Shui Y, Sheng L, Wang K, Hu Q and Wei Q: Epidermal growth factor receptor and human epidermal growth receptor 2 expression in parotid mucoepidermoid carcinoma: Possible implications for targeted therapy. Oncol Rep 19: 435-440, 2008.

8. Ettl T, Schwarz S, Kleinsasser N, Hartmann A, Reichert TE and Driemel O: Overexpression of EGFR and absence of C-KIT expression correlate with poor prognosis in salivary gland carcinomas. Histopathology 53: 567-577, 2008.

9. Senft E, Lemound J, Stucki-Koch A, Gellrich NC, Kreipe H and Hussein K: Expression of cyclin-dependent kinase inhibitor 2A 16, tumour protein 53 and epidermal growth factor receptor in salivary gland carcinomas is not associated with oncogenic virus infection. Int J Oral Sci 7: 18-22, 2015.

10. Pei L and Melmed S: Isolation and characterization of a pituitary tumor-transforming gene (PTTG). Mol Endocrinol 11: 433-441, 1997.

11. Zou H, McGarry TJ, Bernal T and Kirschner MW: Identification of a vertebrate sister-chromatid separation inhibitor involved in transformation and tumorigenesis. Science 285: 418-422, 1999.

12. Jallepalli PV, Waizenegger IC, Bunz F, Langer S, Speicher MR, Peters JM, Kinzler KW, Vogelstein B and Lengauer C: Securin is required for chromosomal stability in human cells. Cell 105: 445-457, 2001

13. Solbach C, Roller M, Fellbaum C, Nicoletti M and Kaufmann M PTTG mRNA expression in primary breast cancer: A prognostic marker for lymph node invasion and tumor recurrence. Breast 13 : $80-81,2004$
14. Wen CY, Nakayama T, Wang AP, Nakashima M, Ding YT, Ito M, Ishibashi H, Matsuu M, Shichijo K and Sekine I: Expression of pituitary tumor transforming gene in human gastric carcinoma. World J Gastroenterol 10: 481-483, 2004.

15. Honda S, Hayashi M, Kobayashi Y, Ishikawa Y, Nakagawa K and Tsuchiya E: A role for the pituitary tumor-transforming gene in the genesis and progression of non-small cell lung carcinomas. Anticancer Res 23: 3775-3782, 2003.

16. Zhou C, Liu S, Zhou X, Xue L, Quan L, Lu N, Zhang G, Bai J, Wang Y, Liu Z, et al: Overexpression of human pituitary tumor transforming gene (hPTTG), is regulated by beta-catenin/TCF pathway in human esophageal squamous cell carcinoma. Int J Cancer 113: 891-898, 2005

17. Ramaswamy S, Ross KN, Lander ES and Golub TR: A molecular signature of metastasis in primary solid tumors. Nat Genet 33 49-54, 2003.

18. Shibata Y, Haruki N, Kuwabara Y, Nishiwaki T, Kato J, Shinoda N, Sato A, Kimura M, Koyama H, Toyama T, et al: Expression of PTTG (pituitary tumor transforming gene) in esophageal cancer. Jpn J Clin Oncol 32: 233-237, 2002.

19. Livak KJ and Schmittgen TD: Analysis of relative gene expression data using real-time quantitative PCR and the 2(-Delta Delta C(T)) Method. Methods 25: 402-408, 2001.

20. Li Z, Tian T, Lv F, Chang Y, Wang X, Zhang L, Li X, Li L, Ma W, Wu J, et al: Six 1 promotes proliferation of pancreatic cancer cells via upregulation of cyclin D1 expression. PLoS One 8: e59203, 2013.

21. Szymczak-Workman AL, Vignali KM and Vignali DA: Verification of 2A peptide cleavage. Cold Spring Harb Protoc 2012: 255-257, 2012.

22. Szymczak AL, Workman CJ, Wang Y, Vignali KM, Dilioglou S, Vanin EF and Vignali DA: Correction of multi-gene deficiency in vivo using a single 'self-cleaving' $2 \mathrm{~A}$ peptide-based retroviral vector. Nat Biotechnol 22: 589-594, 2004.

23. Heaney AP, Singson R, McCabe CJ, Nelson V, Nakashima M and Melmed S: Expression of pituitary-tumour transforming gene in colorectal tumours. Lancet 355: 716-719, 2000.

24. Wen CY, Nakayama T, Wang AP, et al: Expression of pituitary tumor transforming gene in human gastric carcinoma. World J Gastroenterol 10: 481-483, 2004.

25. Ramaswamy S, Ross KN, Lander ES and Golub TR: A molecular signature of metastasis in primary solid tumors. Nat Genet 33: 49-54, 2003. 